\title{
ROLLING OF A RIGID BALL ON A HORIZONTAL DEFORMABLE SURFACE
}

\author{
MAURICE N. BREARLEY' ${ }^{1}$ and NEVILLE J. DE MESTRE ${ }^{2}$
}

(Received 6 January, 2004; revised 23 June, 2004)

\begin{abstract}
The rolling of a ball on a horizontal deformable surface was investigated under the assumptions that the ball was a rigid sphere and the surface was elastic. Finite strain theory was used to develop theoretical results which were found to match observations well in cases where the ball and surface involved were such as to ensure no slipping at the region of contact, including a lawn bowl rolling on a grass rink and a billiard ball rolling on carpet. The theory did not match well the behaviour of a golf ball on a grass green because the ball was too light to enforce the no-slipping condition.
\end{abstract}

\section{Introduction}

Experiments were performed with a billiard ball rolling on carpet, a lawn bowl rolling on a grass rink, and a golf ball rolling on a grass putting green. In each case the ball was launched from an inclined green. In each case the ball was launched from an inclined ramp, and accurate measurements were made of a number of distances travelled at measured times throughout each run.

In every case the rolling surface was plane and horizontal. Under the very rapidly changing stresses induced by a rolling ball, the behaviour of the surface approximates to that of an elastic solid. It is reasonable to assume that the rigidity $\mu$ of the surface is constant and that inertial forces induced in it are negligible. Calculations showed that, in the situations considered, air resistance to the motion of the ball was small enough to be neglected.

Finite strain theory of an elastic solid was found to be adequate to account for the observed nonlinear nature of the deceleration of the ball as a function of time. The

\footnotetext{
185 Dandarriga Drive, Clifton Springs, VIC 3222, Australia.

${ }^{2}$ Faculty of Information Technology, Bond University, Gold Coast, Qld 4229, Australia; e-mail: neville_de_mestre@bond.edu.au.
}

(C) Australian Mathematical Society 2004, Serial-fee code 1446-1811/04 
resulting theory was found to match closely the results observed for both a billiard ball on carpet and a lawn bowl on grass. This was not so, however, for a golf ball on grass; this was because the light golf ball did not enforce a no-slip condition over the region of contact in the way that the heavier balls did.

The approach used here differs from that used by most investigators of this subject. The relevant elasticity theory is shown to lead to a nonlinear differential equation governing the deceleration of the ball. The problem is thus reduced to solving this equation and evaluating the constants which occur in it and in its solution. (For other approaches to the subject, see Bueche and Flom [4], which also contains a list of sixteen other references.)

\section{The experimental arrangements}

It was necessary to measure the distances $x$ travelled by the ball, and the corresponding times $t$, at a number of locations spaced along the path, including the end position. For the billiard ball on carpet this was achieved by recording the progress of the ball by a video camera which was mounted on rails to enable it to move with the ball. A zoom lens enabled accurate distance readings to be made every 0.04 seconds from a metric tape laid beside the path of the ball. It was found that the distances recorded every 0.08 seconds were adequate for analytic purposes.

For the golf ball and lawn bowl the outdoor situation made the use of a moving video camera difficult. Instead, a multi-recording electronic stop-watch was used to record the times for the ball to reach several measured locations, including the end point, the distances being given by a metric tape laid beside the path.

Because only the distances travelled by the bowl along its path were needed in this investigation, the bias causing a curved path was offset by two 20 cent coins taped to the side of the bowl. This was found to be ideal for producing a straight path. (The effect of bias on the path of a bowl has been described elsewhere, see for example Brearley and Bolt [2], Brearley [1].)

All of the balls were launched down inclined ramps. For the billiard ball on carpet, a short aluminium ramp was used, with a curve at the bottom to ensure smooth transition to the carpet. The lawn bowl and golf ball were launched from a 2 metre long wooden ramp of $30^{\circ}$ angle of inclination. A curved steel plate at the foot of the ramp gave smooth access to the grass surface. A number of different starting positions were marked along the ramp to enable different velocities to be attained by the ball at the foot of the ramp.

The vertical heights of the balls were measured at each starting position, and from these their velocities at the bottom of the ramps could be determined. Calculations showed that air resistance had a negligible effect on the motion, and that the lawn 
bowl was close enough to spherical for it to be treated as a sphere when applying the principle of conservation of energy to its motion down the ramp. For the billiard ball and lawn bowl, let

$$
\begin{aligned}
& h=\text { the vertical starting height of the ball on the ramp and } \\
& V=\text { the velocity of the ball on reaching the foot of the ramp. }
\end{aligned}
$$

Then (in obvious notation) the mechanical energy conservation principle gives

$$
\frac{1}{2} m V^{2}+\frac{1}{2}\left(\frac{2}{5} m r^{2}\right)\left(\frac{V}{r}\right)^{2}=m g h \text {. }
$$

Using $g=9.8 \mathrm{~ms}^{-2}$, with $h$ expressed in metres, this gives

$$
V=3.74166 \sqrt{h} \mathrm{~ms}^{-1} \text {. }
$$

This formula was used to calculate the initial velocity of the bowl and billiard ball in each experiment.

Golf balls are not homogeneous as they have dense inner cores surrounded by less dense cores and light urethane covers, making (2.1) and (2.2) inappropriate. The matter will be discussed further in Section 11.

For the experiments on grass, every run from each height $h$ was performed several times, the results compared, and the means of the observed times calculated, with the object of reducing experimental errors. Between each run the ramp was moved sideways by several centimetres so as to prevent the ball from running over a track which had been compressed by a previous run.

\section{The response of the deformable surface}

The ball is assumed to be rigid, its retardation being caused by its deformation of the elastic rolling surface. It is assumed that there is no slipping between the ball and the rolling surface.

The retarding force provided by the surface consists of two parts. One is the result of the ball being in a shallow depression caused by its weight; it is virtually constant, and will be denoted by $F_{1}$. The other part, denoted by $F_{2}$, is induced by the elastic strain caused in the surface by contact with the ball. Since air resistance is neglected, the equation of motion of the ball in the forward direction is

$$
m \dot{v}=-F_{1}-F_{2},
$$

where $m$ is the mass of the ball and $v$ is the velocity of its centre of mass $G$. The form of $F_{2}$ will be determined on the assumption that the rolling surface obeys the laws of elastic finite strain theory. 
Because there is no slipping between the ball and the surface at any point of the region $S$ of contact between them, and the displacements of the surface caused by the ball are very small, the path of every point $P$ on the ball is very nearly cycloidal, as shown in Figure 1 (a).

The position of the ball will be referred to rectangular axes $O x y$, with $O x$ in the direction of motion and lying in the surface on which the ball is rolling, as in Figure 1.

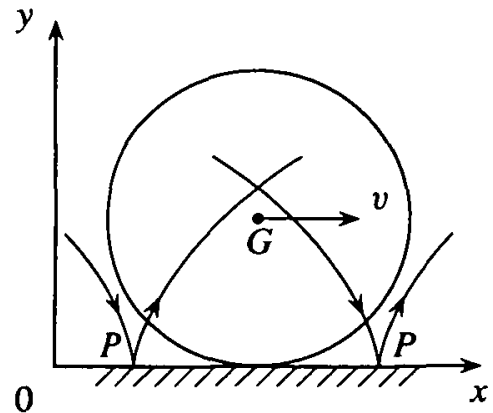

(a)

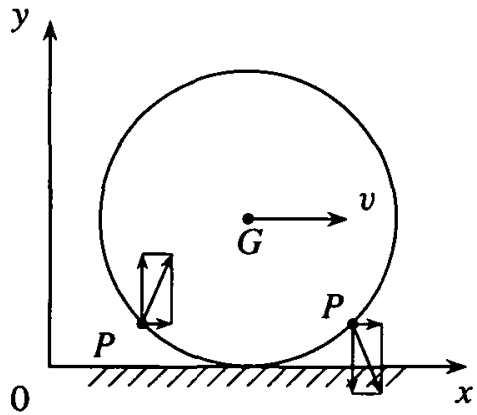

(b)

FIGURE 1. Cycloidal paths and velocity components of points of the rolling ball.

The cycloidal path of $P$ has horizontal and vertical components of velocity as shown in Figure 1 (b). When $P$ is in contact with the surface on which the ball is rolling, it induces in it a shear strain which is very nearly horizontal because the depression is small, and a compression strain which is very nearly vertical. In the following section the horizontal strain will be calculated. From this the associated stress can be found, and when summed over all points of $S$ it will be the reverse of the force $F_{2}$ acting on the ball.

At the end of the travel of the ball, the forces $F_{1}$ and $F_{2}$ drop to zero, as they are caused purely by the motion of the ball. This will not occur as finite discontinuities of the forces, but as very sharp declines accompanying relaxation of the strains of the deformed surface.

\section{The stress-strain relations for the surface}

Every vertical "slice" of the ball parallel to the plane $O x y$ in Figure 1, which has a circumference making contact with the region $S$, is moving only in a direction parallel to $O x y$. Each such slice can therefore reasonably be expected to cause stresses and strains which have no components perpendicular to $O x y$. The elasticity problem involved is thus one known as "plane strain", with no dependence on a third space dimension perpendicular to $O x y$. 


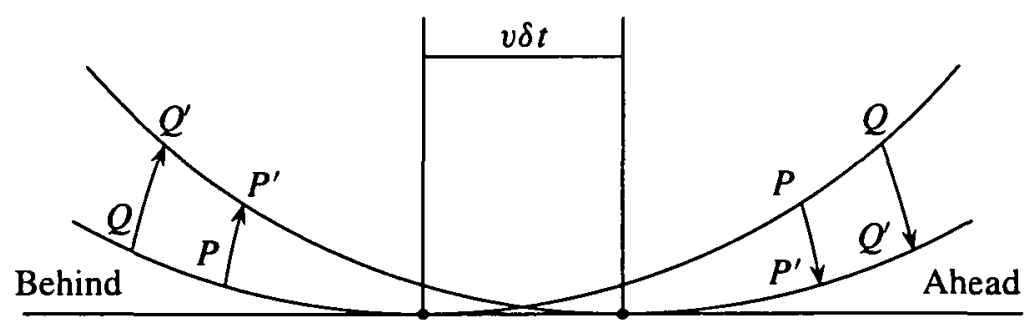

FIGURE 2. Movements of points $P, Q$ to $P^{\prime}, Q^{\prime}$ during strain.

It is convenient to rename the axes $O x y$ as $O x_{1} x_{2}$. The general form of the stressstrain relations is (Sokolnikoff [6])

$$
p_{i j}=\lambda \theta \delta_{i j}+2 \mu e_{i j}
$$

where $\lambda, \mu$ are Lamé parameters and $\theta$ is the dilatation. There are grounds for believing that finite strain theory is appropriate, for which the form of the strain tensor is

$$
e_{i j}=\frac{1}{2}\left(\frac{\partial u_{j}}{\partial x_{i}}+\frac{\partial u_{i}}{\partial x_{j}}\right)-\frac{1}{2} \frac{\partial u_{k}}{\partial x_{i}} \frac{\partial u_{k}}{\partial x_{j}},
$$

where the $u_{i}$ are displacements, and summation over $k$ is intended in the last term.

The shear stress of interest in (4.1) is $p_{21}=2 \mu e_{21}$, since this is responsible for the shear force

$$
F_{2}=\iint_{S} p_{21} d S=2 \mu \iint_{S} e_{21} d S,
$$

where the integration is over the whole region $S$ of contact.

\section{Consideration of the surface displacements}

It is necessary to consider separately the cases when the points under strain are ahead of and behind the lowest point of the ball, as shown in Figure 2.

Figure 2 depicts the movement of the bottom of the ball during a small time interval $\delta t$, in the course of which the centre moves a distance $v \delta t$. The displacements $P P^{\prime}$ and $Q Q$ experienced by points $P$ and $Q$ during $\delta t$ are shown for the cases in which the points are ahead of and behind the lowest point of the ball.

Before considering these displacements in detail it can be shown that they are proportional to the ball velocity $v$. Writing $v \delta t=d$ for brevity, the situation involved may be represented by Figure 3. 


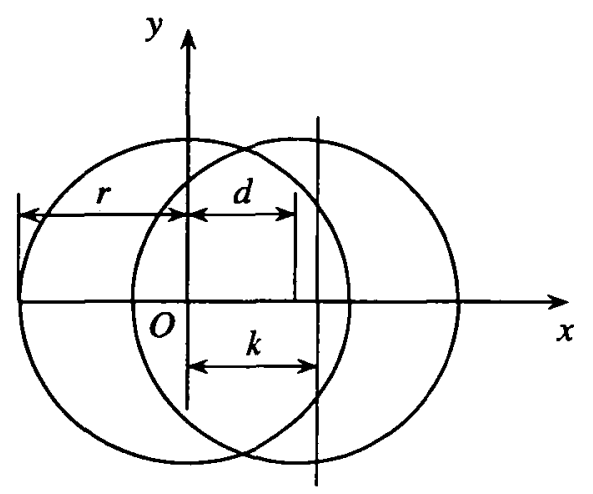

FIGURE 3. Two successive ball positions a distance $d$ apart.

Let the equations with respect to the axes Oxy of the circular cross-sections of the ball shown in Figure 3 be

$$
x^{2}+y^{2}=r^{2}, \quad(x-d)^{2}+y^{2}=r^{2} .
$$

The two circles intersect the vertical line with equation $x=k$ at values of $y$ given by

$$
y=\left(r^{2}-k^{2}\right)^{1 / 2}, \quad y=\left[r^{2}-(k-d)^{2}\right]^{1 / 2}
$$

respectively. Although not shown in Figure 3, it is intended that $d \ll k \ll r$. It is easily seen that the difference between the values of $y$ given by the last two equations is approximately $k d / r=k v \delta t / r$. When interpreted in the context of Figure 2 this means that the small displacements $P P^{\prime}$ and $Q Q^{\prime}$ are proportional to $v$.

To consider these displacements it is desirable to enlarge the relevant parts of Figure 2, as shown in Figure 4.

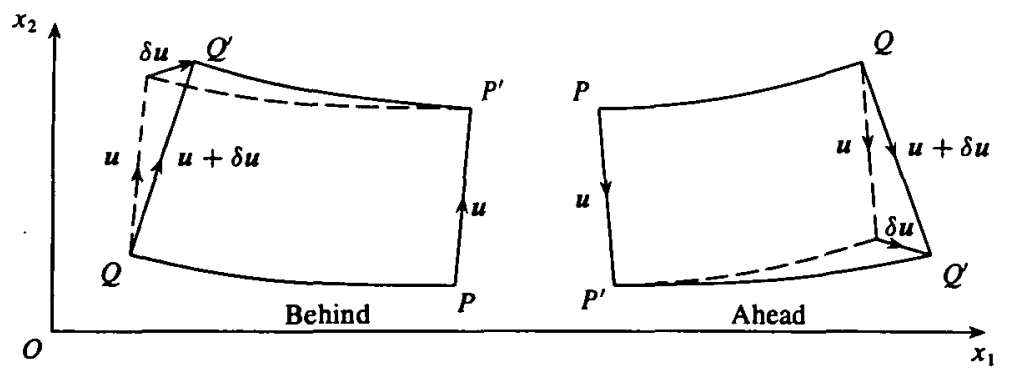

FIGURE 4. Displacements at points $P, Q$ ahead of and behind the lowest point of the ball.

The displacements of $P$ and $Q$ are shown in vector notation in Figure 4. The components of $\delta u$ with respect to the axes $O x_{1} x_{2}$ are denoted as $\delta u=\left(\delta u_{1}, \delta u_{2}\right)$. 
If the coordinates of $P$ are $\left(x_{1}, x_{2}\right)$, and those of $Q$ are $\left(x_{1}+\delta x_{1}, x_{2}+\delta x_{2}\right)$, then $\mathbf{P Q}=\left(\delta x_{1}, \delta x_{2}\right)$. From Figure 4 it can be seen that $\left|\delta x_{1}\right| \gg\left|\delta x_{2}\right|$ for points within the small region $S$ of contact. It is also clear that:

behind the ball: $\delta u_{1}>0, \delta u_{2}>0, \delta x_{1}<0, \delta x_{2}>0$;

ahead of the ball: $\delta u_{1}>0, \delta u_{2}<0, \delta x_{1}>0, \delta x_{2}>0$.

\section{The surface strain and stress}

From (4.2) we have

$$
e_{21}=\frac{1}{2}\left(\frac{\partial u_{1}}{\partial x_{2}}+\frac{\partial u_{2}}{\partial x_{1}}\right)-\frac{1}{2}\left(\frac{\partial u_{1}}{\partial x_{2}} \frac{\partial u_{1}}{\partial x_{1}}+\frac{\partial u_{2}}{\partial x_{2}} \frac{\partial u_{2}}{\partial x_{1}}\right) .
$$

From the work in Section 5 it follows that the magnitudes of all the partial derivatives in (6.1) are proportional to $v$. Also in the first term on the right-hand side of (6.1), the derivative $\partial u_{1} / \partial x_{2}$ is the dominant one, and this term is positive both ahead of and behind the ball. Hence

$$
\frac{1}{2}\left(\frac{\partial u_{1}}{\partial x_{2}}+\frac{\partial u_{2}}{\partial x_{1}}\right)=f_{1} v
$$

where $f_{1}$ is a function of position in the contact area $S$ which is positive throughout $S$.

From Section 5 it also follows that the magnitude of the second term on the righthand side of (6.1) is proportional to $v^{2}$, and that its sign changes on moving from behind the ball to ahead of it. So

$$
-\frac{1}{2}\left(\frac{\partial u_{1}}{\partial x_{2}} \frac{\partial u_{1}}{\partial x_{1}}+\frac{\partial u_{2}}{\partial x_{2}} \frac{\partial u_{2}}{\partial x_{1}}\right)= \pm f_{2} v^{2}
$$

where $f_{2}$ is a positive function of position in $S$, and the sign is + behind the ball and - ahead of it.

The form of (6.3) suggests that its contributions to the strain $e_{21}$ would annul one another when summed over the whole of $S$. The deformations in the rolling surface are, however, not symmetrical about the lowest point of the ball. The ball compresses the region ahead of it, increasing the magnitudes of all four positive derivatives in the last term in (6.1); and behind the ball the strain is reduced by the ball's progress, thus reducing in this region the magnitudes of both negative products in the last term in (6.1). The net result is a negative value when the last term (including its coefficient $-1 / 2)$ is integrated over the whole of the region $S$.

On using (6.1)-(6.3), (4.3) yields

$$
F_{2}=2 \mu \iint_{S}\left(f_{1} v \pm f_{2} v^{2}\right) d S=A_{1} v-A_{2} v^{2}
$$


where $A_{1}$ and $A_{2}$ are positive constants. Their values will, of course, depend on the size and weight of the ball and on the character of the rolling surface. Substitution from (6.4) in (3.1) yields $m \dot{v}=-F_{1}-\left(A_{1} v-A_{2} v^{2}\right)$, whence

$$
\dot{v}=-a-b v+c v^{2}
$$

where $a, b, c$ are positive constants. This is the equation governing the deceleration of the ball.

\section{The solution of the deceleration equation}

In terms of the distance $x$ travelled in time $t,(6.5)$ is

$$
\ddot{x}=-a-b \dot{x}+c \dot{x}^{2} .
$$

The solution of (7.1) is needed under the initial conditions

$$
t=0, \quad x=0, \quad \dot{x}=V,
$$

and is shown in Appendix A to be

$$
x=K t-c^{-1} \ln \left[1+F e^{-D t}\right]+G,
$$

where

$$
\begin{aligned}
& c K^{2}-b K-a=0, \\
& D=b-2 K c \\
& F=\frac{c(V-K)}{D-c(V-K)} \\
& G=c^{-1} \ln (F+1) .
\end{aligned}
$$

These equations show that the values of $K$ and $D$ are the same for all initial velocities $V$, but that the values of $F$ and $G$ vary with $V$.

A method of calculating the values of the constants in the equation of motion (7.1) and in the solution (7.2) from the results of experimental runs of the ball is described in detail in Appendix B. It requires knowing the values of the initial velocity $V$ and the total time $T$ and distance $X$ for three different runs. Experience shows that more accurate results are obtained by using results from a single run to calculate the values of the constants, rather than from three entirely different runs. To obtain the three data triples $V, T, X$ necessary for evaluation of the constants, the following device is used.

The longest run is chosen because any errors in measuring its parameters will be proportionally smaller than for shorter runs. Let $V_{1}, T_{1}, X_{1}$ denote its measured initial 
velocity and observed total time and distance. A graph can be drawn carefully through all corresponding time and distance pairs $(t, x)$ observed during the run, including $(0,0)$ and $\left(T_{1}, X_{1}\right)$.

An intermediate point on this graph is selected, at which the coordinates, $\left(t_{2}, x_{2}\right)$ say, can be read off. The slope of the graph at this point is determined carefully; its magnitude represents the initial velocity $V_{2}$ of an intermediate "run", for which the total time and distance are $T_{2}=T_{1}-t_{2}$ and $X_{2}=X_{1}-x_{2}$.

The process is then repeated for another selected intermediate point on the graph, having measured coordinates $\left(t_{3}, x_{3}\right)$. The measured slope there represents the initial velocity $V_{3}$ of another intermediate "run", for which the total time and distance are $T_{3}=T_{1}-t_{3}$ and $X_{3}=X_{1}-x_{3}$.

In this way the magnitudes $V, T, X$ for three "runs" are obtained, enabling the values of the constants in the solution to be found. The process will be illustrated in the following sections.

\section{Lawn bowl rolling on a grass rink}

Experiments were performed with a lawn bowl of diameter 5 inches $(=0.127 \mathrm{~m})$ on a grass rink. The mass of the bowl (including the two 20 cent coins taped to its side to counteract the bias and make it run straight) was $1.573 \mathrm{~kg}$. This is large enough to ensure that no slipping could occur between the bowl and the grass, so the theory expounded in the previous sections should be applicable. The rink was dry, with newly mown grass, and was classed as one of medium speed.

Runs were made from four different starting heights on the ramp. Equation (2.2) enabled the initial velocity $V$ on the grass to be calculated in each case. Table 1 shows some of the data, including the intermediate times $t_{n}$ and distances $x_{n}$, total times $T$ and total distances $X$ recorded by stop-watch and measuring tape. The times and total distances are the averages of those recorded over several trials. The intermediate distances $x_{n}$ were indicated by pieces of wooden dowel laid on the grass at right angles to the measuring tape. The run numbers are those marked on the ramp at the different starting heights $h$. The times are in seconds and the distances in metres.

In all of the numerical work, the units are metric and will usually not be stated. The number of figures carried in many parameters is for purposes of calculation only, and is not indicative of the accuracy with which their values are known.

Three sets of data were obtained from Run 1 by the method described in Section 7 , using $\left(t_{2}, x_{2}\right)$ and $\left(t_{4}, x_{4}\right)$ as the intermediate points. The results obtained were $K=-1.08372, c=0.03748$ and $D=0.23351$.

The values of $F$ and $G$ for each run were then calculated from (7.5) and (7.6). The results are as shown in Table 2 . 
TABLE 1. Lawn bowl experimental results.

\begin{tabular}{|cccll|}
\hline Run no. & \multicolumn{1}{c}{1} & \multicolumn{1}{c}{2} & \multicolumn{1}{c}{3} & \multicolumn{1}{c|}{4} \\
\hline$h(\mathrm{~m})$ & 0.867 & 0.681 & 0.491 & 0.281 \\
$V\left(\mathrm{~ms}^{-1}\right)$ & 3.484 & 3.088 & 2.622 & 2.018 \\
$t_{1}$ & 1.935 & 2.26 & 1.19 & 1.014 \\
$x_{1}$ & 6.00 & 6.00 & 3.00 & 2.00 \\
$t_{2}$ & 3.075 & 3.27 & 2.256 & 1.76 \\
$x_{2}$ & 9.00 & 8.00 & 5.00 & 3.00 \\
$t_{3}$ & 4.4175 & 4.436 & 3.548 & 2.637 \\
$x_{3}$ & 12.00 & 10.00 & 7.00 & 4.00 \\
$t_{4}$ & 6.215 & 6.032 & 5.534 & 3.805 \\
$x_{4}$ & 15.00 & 12.00 & 9.00 & 5.00 \\
$t_{5}$ & 9.5625 & & & \\
$x_{5}$ & 18.00 & & & \\
$T$ & 11.02 & 9.81 & 8.31 & 6.51 \\
$X$ & 18.33 & 13.82 & 9.91 & 5.85 \\
\hline
\end{tabular}

TABLE 2. Values of $F$ and $G$ for the lawn bowl.

\begin{tabular}{|ccccc|}
\hline Run no. & 1 & 2 & 3 & 4 \\
\hline$F$ & 2.74752 & 2.02655 & 1.46788 & 0.99372 \\
$G$ & 35.247 & 29.547 & 24.102 & 18.410 \\
\hline
\end{tabular}

The value of $b$ was then found from (7.4), and that of $a$ from (7.3); they are $a=0.209 \mathrm{~ms}^{-2}$ and $b=0.152 \mathrm{~s}^{-1}$.

The numerical forms of the solution (7.2) are now known for all of the four runs investigated. They enable the graphs of $x$ versus $t$ shown in Figure 5 to be plotted. This figure also shows the observed points $(t, x)$ found during the experiments. The agreement between calculated and observed results is excellent, lending support to the theory on which the calculations were based.

\section{The velocity and acceleration of the lawn bowl}

So far it has been tacitly assumed that the acceleration formula (7.1) applies throughout the whole run of the bowl. The acceleration actually undergoes very sharp changes at the start and finish of a run, their durations being so brief that their influences on the path of the bowl are imperceptible. These changes will now be considered, together with the velocity of the bowl, using Run 1 as an illustrative example.

The velocity at any instant is easily found by differentiating the solution (7.2). This 


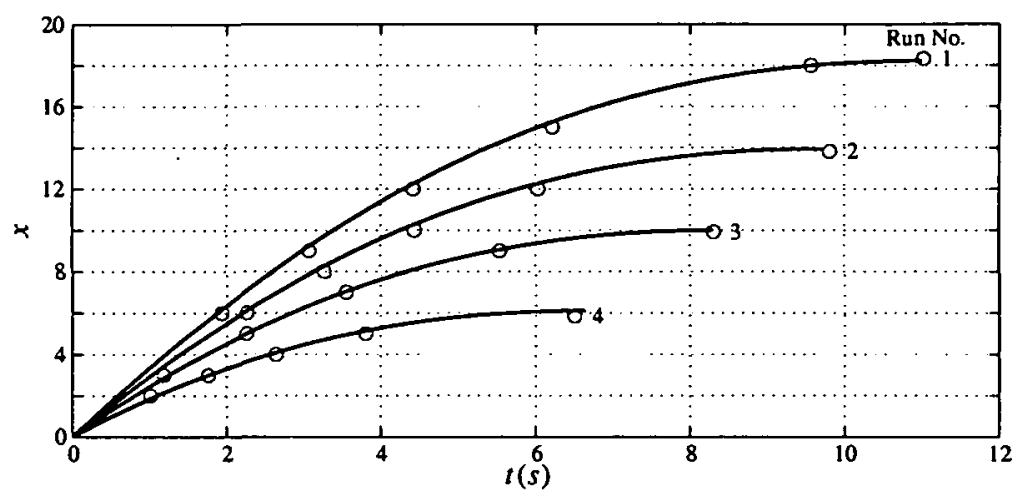

FIGURE 5. Distance $x$ versus time $t$ for lawn bowl on a grass rink.

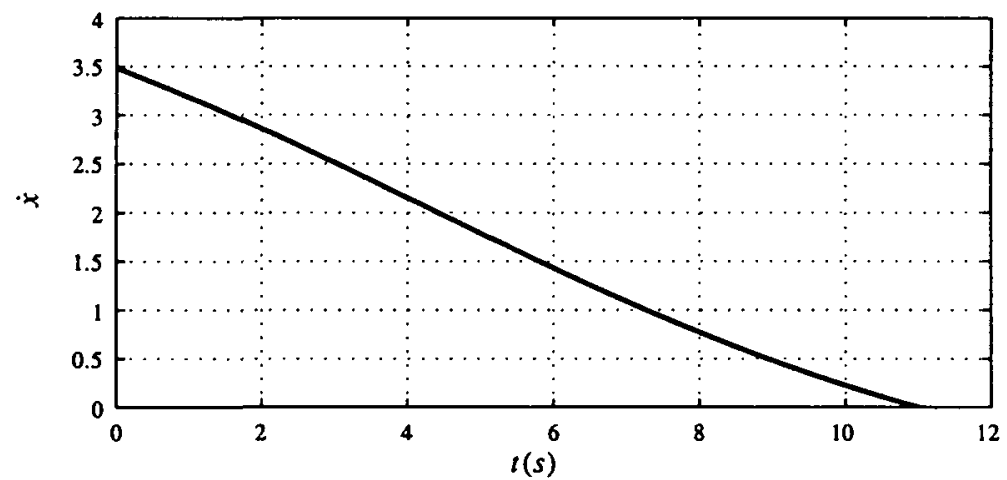

FIGURE 6. Velocity $\dot{x}$ of lawn bowl versus $t$, Run 1 .

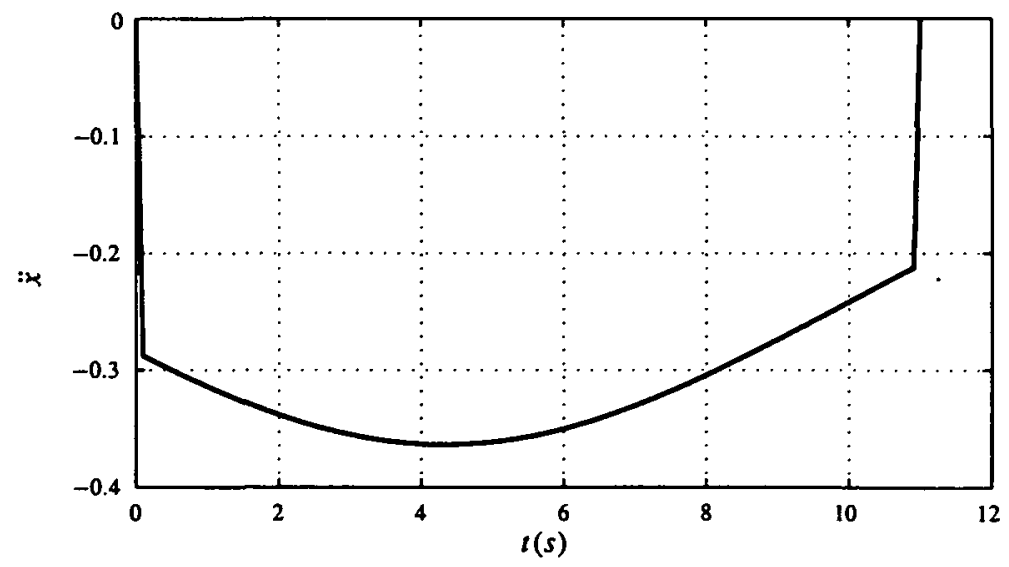

FIGURE 7. Acceleration of lawn bowl versus $t$, Run 1. 
gives the velocity as

$$
\dot{x}=K+c^{-1} D\left[1+F^{-1} e^{D t}\right]^{-1} .
$$

After insertion of the values of the constants found in Section 8, this equation enables the graph of $\dot{x}$ versus $t$ for Run 1 to be drawn in the relevant interval $0 \leq t \leq 11.02$. It is shown in Figure 6.

During its travel down the launching ramp the acceleration of the bowl is constant. The curved metal piece at the foot of the ramp is horizontal at its lower end, which rests on the grass, so the acceleration of the bowl reduces to zero at this point.

On leaving the metal piece the bowl sinks into the grass to a very small depth. It is not hard to show that, if this small depth is estimated to be $1 \mathrm{~mm}$, the duration of the transition of the bowl from metal piece to grass is about 3 milliseconds in the case of Run 1 , for which the initial bowl velocity $V$ is $3.484 \mathrm{~ms}^{-1}$. During this time the acceleration of the bowl decreases sharply from zero to a negative value.

On the grass the deceleration is given by the derivative of $(9.1)$, namely

$$
\ddot{x}=-\frac{e^{D t}}{c F}\left[\frac{D}{1+F^{-1} e^{D t}}\right]^{2}
$$

and this governs the motion of the bowl for most of its run. Very near the end of the run, the horizontal displacements of the surface, which have been induced by the motion of the bowl, are decreasing to zero. The force $F_{2}$ described in Section 6 is therefore decreasing to zero, and the same is true of the force $F_{1}$ which vanishes when the forward movement of the bowl ceases. The acceleration is therefore tending to zero rapidly, rather than undergoing a finite discontinuity where $\dot{x}=0$ as suggested by (7.1). Calculations suggest that the duration of this end phase is of the order of 30 milliseconds.

When the values of the constants appropriate for Run 1 of the bowl are inserted in (9.2) this equation enables the graph of $\ddot{x}$ versus $t$ shown in Figure 7 to be drawn. The slopes of the graph at the start and end have been reduced to make the terminal features visible.

\section{Billiard ball rolling on carpet}

The experimental arrangements described in Section 2 were used for the billiard ball rolling on loop pile carpet. A run was made over only one distance of $241 \mathrm{~cm}$. Because of the shortness of this run, the observations and calculations were all made in centimetre units rather than metric.

Analysis of the results showed that the billiard ball on carpet behaved in the same way as the lawn bowl on grass, in the sense that its deceleration is given by (7.1), so 


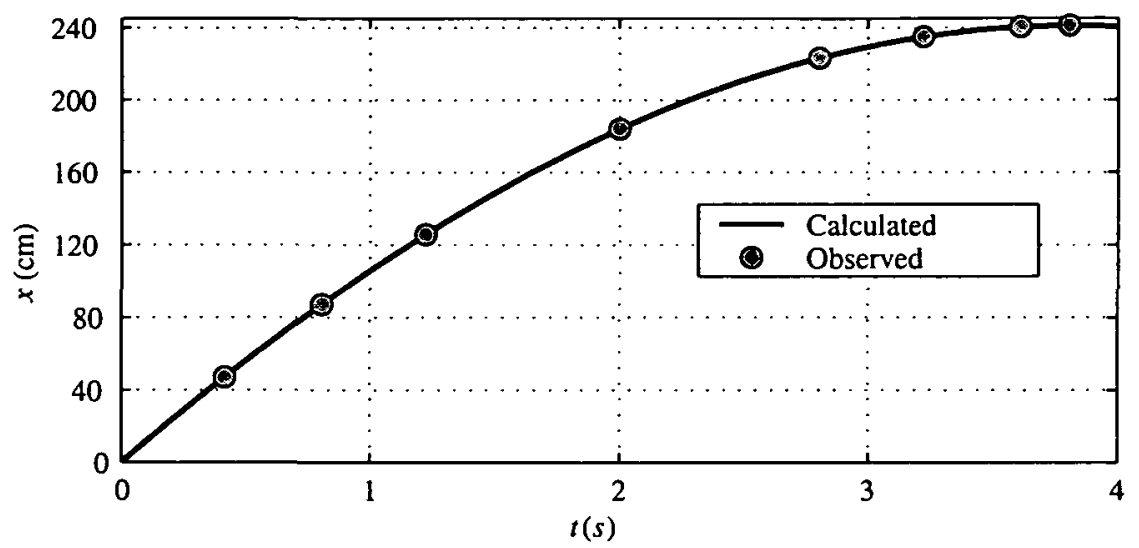

FIGURE 8. Billiard ball on loop pile carpet.

that the solution (7.2) is relevant. With an initial velocity of $V=120.0 \mathrm{~cm} \mathrm{~s}^{-1}$, the form of the solution was found to be

$$
x=-135.49 t-701.70 \ln \left[1+4.1799 e^{-0.44717 t}\right]+1154.2 .
$$

The associated values of the constants in the deceleration equation were found to be $a=34.4 \mathrm{~cm} \mathrm{~s}^{-2}, b=0.0610 \mathrm{~s}^{-1}$ and $c=0.00143 \mathrm{~cm}^{-1}$.

Figure 8 shows the calculated form of the path of the billiard ball predicted by the above solution, and also some of the observed points $(t, x)$. The agreement between calculated and observed results is exceedingly close, lending support to the theory on which the solution was based.

\section{Golf ball rolling on grass}

It is well known that golf balls are not homogeneous but consist of a dense inner core, a less dense outer core and a thin plastic cover. They cannot be treated as homogeneous spheres. Equations (2.1) and (2.2) are therefore inappropriate for a golf ball.

It is possible to calculate by suitable experiments the moment of inertia of the golf ball, and to use it in a conservation equation similar to (2.1). This leads to an equation for the initial velocity $V$ to replace (2.2).

A set of experiments similar to those described in Section 8 for the lawn bowl was carried out with the golf ball on a horizontal putting green of medium speed. The results were used as in Section 8 to calculate theoretical paths for all four runs. The details of the experiments and calculations are shown in an earlier paper (Brearley and de Mestre [3]). 
The agreement between the calculated and observed results was poor, the calculated value of $X$ being $9 \%$ less than the observed value in Run 1, and this deficit increases to $16 \%$ in Run 4 . The reason for this is that the golf ball is too light to ensure the condition of non-slip between it and the surface, on which the theory is based. The light ball skips along on the blades of grass in a way that the massive bowl did not.

The experiments with a golf ball were repeated on the medium speed bowling rink which was used earlier for the lawn bowl (see Section 8). The results were similar to those found on the putting green, though the distances travelled by the ball were greater by about $30 \%$ than those on the green.

\section{Summary and conclusions}

The rolling of a ball on a plane horizontal surface was investigated by treating the ball as rigid and the surface as deformable. Experiments were performed by launching from ramps a lawn bowl on a grass bowling rink, a golf ball on a grass putting green, and a billiard ball on a loop pile carpet.

It was adequate to treat the bowl and billiard ball as homogeneous spheres, but investigations showed that the density of the golf ball varied internally to a degree that required its moment of inertia being evaluated experimentally.

The plane surface was treated as elastic, and finite strain theory was used to derive the form of the retarding force on the rolling ball, on the assumption that there was no slipping at the region of contact with the surface. This enabled the equation of motion of the ball to be formed, and its solution led to a prediction of the distance travelled as a function of time, for any known initial velocity.

For the lawn bowl and billiard ball the agreement between calculated and observed results was excellent, indicating the appropriateness of the theory. Because the golf ball is too light to ensure the no-slip condition on grass, accurate predictions could not be made about its travel distances on this surface.

\section{Appendix A. The solution of the equation of motion}

The equation of motion is

$$
\ddot{x}=-a-b \dot{x}+c \dot{x}^{2},
$$

which is a form of the Riccati equation (Murphy, [5]).

On substituting $y=x-K t$, where

$$
c K^{2}-b K-a=0
$$


and then putting $w=\dot{y}$ it is found that $\dot{w}=c w^{2}-D w$, where

$$
D=b-2 K c \text {. }
$$

Solving by separation of variables yields

$$
w=\frac{D c^{-1}}{1+F^{-1} e^{D t}},
$$

where $F$ is constant.

Since $w(0)=V-K$, where $V=\dot{x}(0)$, we find that

$$
F=\frac{c(V-K)}{D-c(V-K)} \text {. }
$$

Integrating again to find $x$ leads to

$$
x=K t-c^{-1} \ln \left(1+F e^{-D t}\right)+G,
$$

where $G$ is a constant. Since $x(0)=0$, we have

$$
G=c^{-1} \ln (F+1) \text {. }
$$

\section{Appendix B. Evaluation of the constants}

Differentiating the solution (A.5) for $\mathrm{x}$ gives

$$
\dot{x}=K+\frac{c^{-1} F D e^{-D t}}{1+F e^{-D t}} .
$$

This result, and the form (A.4) for $F$, enable $\ln \left(1+F e^{-D t}\right)$ to be eliminated from $x$, giving

$$
(K c+D) t=c x-\ln (\dot{x}-K)+\ln (V-K) .
$$

At the end of a run, we have $t=T, x=X, \dot{x}=0$, and so

$$
(K c+D) T=c X+\ln \left(1-V K^{-1}\right) .
$$

Using data $\left(T_{1}, V_{1}\right),\left(T_{2}, V_{2}\right),\left(T_{3}, V_{3}\right)$ from three runs gives three equations for the three unknowns $K, c, D$. By eliminating $K c+D$ between two pairs of these equations, and then $c$ between the two resulting equations, an equation for $K$ is obtained. This can be solved numerically by Newton's method.

The constants $c$ and $D$ can then be found, after which (A.3) yields the value of $b$, and then (A.2) gives $a$. These constants depend only on the properties of the ball and the surface.

For a given run, the constants $F$ and $G$ can be found from (A.4) and (A.6), their values depending on the initial velocity $V$. The function $x(t)$ then gives values which can be compared with experimental results for selected values of $t$. 


\section{Acknowledgements}

The writers are grateful to the staff of the Audio Visual Services at Bond University for assistance with the billiard ball experiments, and to the Presidents of the Boomerang Park Golf Club and Mudgeeraba Bowls Club on the Gold Coast, Queensland, for making their facilities available for experiments.

\section{References}

[1] M. N. Brearley, "The motion of a biased bowl with perturbing projection conditions", Proc. Camb. Phil. Soc. 57 (1961) 131-151.

[2] M. N. Brearley and B. A. Bolt, "The dynamics of a bowl", Quart. J. Mech. Appl. Math. 11 (1958) 351-363.

[3] M. N. Brearley and N. J. de Mestre, "How do lawn bowls and golf balls slow down on grass?", Proc. 6th Maths and Computers in Sport Conference (2002) 78-91.

[4] A. M. Bueche and D. G. Flom, "Surface friction and dynamic mechanical properties of polymers", Wear 2 (1958/59) 168-182.

[5] G. M. Murphy, Ordinary differential equations and their solutions (Van Nostrand, New York, 1960).

[6] I. S. Sokolnikoff, Mathematical theory of elasticity, 2nd ed. (McGraw-Hill, New York, 1956). 